\title{
OPEN The aptamer BT200 blocks von Willebrand factor and platelet function in blood of stroke patients
}

\author{
Katarina D. Kovacevic ${ }^{1 凶}$, Stefan Greisenegger ${ }^{2}$, Agnes Langer ${ }^{2}$, Georg Gelbenegger ${ }^{1}$, \\ Nina Buchtele ${ }^{3}$, Ingrid Pabinger ${ }^{4}$, Karin Petroczi ${ }^{1}$, Shuhao Zhu ${ }^{5}$, James C. Gilbert ${ }^{5}$ \& \\ Bernd Jilma ${ }^{1}$
}

The effect of conventional anti-platelet agents is limited in secondary stroke prevention, and their effects are blunted under high shear stress in the presence of increased levels of circulating von Willebrand factor (VWF). VWF is critically involved in thrombus formation at sites of stenotic extracranial/intracranial arteries. A third generation anti-VWF aptamer (BT200) has been generated which could be useful for secondary stroke prevention. To characterize the effects of BT200 in blood of patients with large artery atherosclerosis stroke (LAA). Blood samples were obtained from 33 patients with acute stroke or transient ischemic attack to measure inhibition of VWF activity and VWF-dependent platelet function. Patients who received clopidogrel or dual antiplatelet therapy did not differ in VWF dependent platelet function tests from aspirin treated patients. Of 18 patients receiving clopidogrel with or without aspirin, only 3 had a prolonged collagen adenosine diphosphate closure time, and none of the patients had ristocetin induced aggregation in the target range. BT200 concentration-dependently reduced median VWF activity from 178 to $<3 \%$, ristocetin induced platelet aggregation from $40 \mathrm{U}$ to $<10 \mathrm{U}$ and prolonged collagen adenosine diphosphate closure times from $93 \mathrm{~s}$ to $>300 \mathrm{~s}$. Baseline VWF activity correlated $(r=0.86, p<0.001)$ with concentrations needed to reduce VWF activity to $<20 \%$ of normal, indicating that BT200 acts in a target concentrationdependent manner. Together with a long half-life supporting once weekly administration, the safety and tolerability observed in an ongoing phase I trial, and the existence of a reversal agent, BT200 is an interesting drug candidate.

The burden of stroke on our healthcare delivery system continues to grow as our population ages and the incidence and prevalence of stroke rises. In the US, each year $\sim 795,000$ people experience a new or recurrent stroke. Between 2015 and 2035, total direct medical stroke-related costs are projected to more than double, from $\$ 36.7$ billion to $\$ 94.3$ billion $^{1}$. Globally, the problem is even larger. In China, for example, stroke is now the leading cause of death despite advances in underlying risk factor management. Furthermore, stroke event rates and stroke-related mortality rates do not capture the full impact on society. Since a non-fatal stroke is often disabling, stroke-related Disability Adjusted Life Years lost is on par with that related to ischemic heart disease ${ }^{2}$.

Many of the antiplatelet drugs are effective and useful in coronary disease but when it comes to the cerebral vascular diseases or stroke, heparin ${ }^{3,4}$ and antiplatelet drugs, GPIIb/IIIa inhibitors like abciximab ${ }^{5}$ or tirofiban $^{6,7}$, $\mathrm{P} 2 \mathrm{Y} 12$ blockers such as prasugrel ${ }^{8}$ or the thrombin-receptor antagonist vorapaxar ${ }^{9}$ cause treatment-induce bleedings and they are contraindicated in stroke. Current secondary prevention of non-cardioembolic stroke consists of aspirin or clopidogrel alone, or aspirin in combination with dipyridamole or clopidogrel in some circumstances, and there is an obvious need for new and improved anti-thrombotic therapy in this field.

BT200 is a third-generation anti- Von Willebrand factor (VWF) aptamer ${ }^{10}$ which inhibits the VWF A1 domain and therefore inhibits the VWF-GPIb axis. VWF is a large glycoprotein and plays a major role in thrombus formation ${ }^{11}$ : It forms large functional multimers that can elongate to long strings that capture platelets in cases of moderate to high shear forces ${ }^{12-15}$. Because VWF is a key factor for the platelet plug formation at high shear rates, other platelet inhibitors such as GPIIb/IIIa (abciximab), P2Y12 (clopidogrel, ticagrelor, prasugrel

${ }^{1}$ Department of Clinical Pharmacology, Medical University of Vienna, Währinger Gürtel 18-20, 1090 Vienna, Austria. ${ }^{2}$ Department of Neurology, Medical University of Vienna, Vienna, Austria. ${ }^{3}$ Department of Internal Medicine I, Medical University of Vienna, Vienna, Austria. ${ }^{4}$ Division of Hematology, Department of Internal Medicine I, Medical University of Vienna, Vienna, Austria. ${ }^{5}$ Guardian Therapeutics, Lexington, MA, USA. ${ }^{\square}$ email: katarina.kovacevic@meduniwien.ac.at 
or cangrelor) or cyclooxygenase-1 (aspirin) are less potent when VWF levels are increased ${ }^{16-18}$. As VWF is a driver $^{19}$ and an independent predictor of stroke recurrence ${ }^{20}$ and mortality ${ }^{21,22}$ and there is a need for improved stroke therapy, we wanted to explore the concentration-effect curves of BT200 in the blood of stroke patients. We hypothesized that BT200 concentration-dependently inhibits platelet function ex vivo and that concentrations needed to inhibit VWF will be target concentration-dependent.

\section{Methods}

This was a non-interventional study performed by using in vitro assays on blood samples collected from 33 patients suffering from stroke due to Large Artery Atherosclerosis (LAA), who provided written informed consent after Ethics Committee approval. Inclusion criteria for patients were that they were older than 18 years, able to give informed consent; they had an established diagnosis of extra- or intra-cranial, large vessel cerebrovascular disease and history of TIA/stroke or a carotid revascularization procedure. Patients were excluded if they were currently treated with unfractionated heparin, warfarin, or a Novel Oral Anti-Coagulant (e.g. rivaroxaban, apixaban, dabigatran, etc.).

The study protocol was designed in accordance with the Declaration of Helsinki and approved by the Ethics Committee of the Medical University of Vienna. Patients were recruited at the Medical University of Vienna and Vienna General Hospital department of Neurology. Blood samples were collected from an antecubital vein via a butterfly venipuncture device and transferred to the appropriate blood collection container either to be analyzed immediately or to be centrifuged for subsequent analysis.

All assays were conducted in the laboratory of the Department of Clinical Pharmacology. In order to characterize the concentration-effect relationship for BT200 in a set of different assays thought to be specifically related to VWF function, 4 assays that are specifically designed to measure the binding of VWF via its A1 domain to platelet GPIb were performed. The first analysis was done with Platelet function analyzer PFA-100 (Dade Behring) - an approved medical device normally used for the diagnosis of deficiency of VWF in whole-blood, a functional assay that quantifies the rate at which a platelet "plug" can form under shear stress in a VWFdependent process. We have measured collagen adenosine diphosphate (CADP-CT) induced closure time ${ }^{23}$. The second analysis was done with Multiple Electrode Aggregometry (MEA) (Multiplate). Ristocetin was used as an agent to measure ristocetin induced aggregation time ${ }^{24}$, this test is sensitive to congenital as well as acquired VWF deficiency ${ }^{25}$. The third analysis was VWF:RCo, it is a standard clinical laboratory assay that quantifies the activity of VWF in the patient's sample via agglutination of donor platelets, using added ristocetin to circumvent the physiologic requirement for the shear force to activate $\mathrm{VWF}^{26}$. The fourth analysis was REAADS VWF activity, it is an enzyme immunoassay that specifically recognizes the portion of VWF which binds to platelets (A1 domain $)^{27}$. For each of these assays, a series of spiked-in concentrations of BT200 from 0.1 to $15 \mu \mathrm{g} / \mathrm{mL}$ were tested.

We used ROTEM thromboelastometry (TemInnovations) to rule out an undue interference with the coagulation system, it measures clotting times (CT and CFT). No activator was used but samples were only recalcified ${ }^{28}$.

Patients with average VWF plasma levels of $24 \%$ that of normal had a $35-67 \%$ reduced risk for ischemic stroke as compared to controls, suggesting that partial inhibition of VWF could be protective in a stroke-prone population ${ }^{29}$ which is why we aimed to reach $<20 \%$ of normal VWF activity. Two-sided $\mathrm{p}$-values of $\mathrm{p}<0.05$ were considered significant.

Statistical analysis. Data were analyzed descriptively. Non-parametric tests were applied as appropriate. We used a Kruskal Wallis test and the U-test to compare the lowest concentrations that will maximally prolong CADP-CT or reduce ristocetin induced aggregation to $<20 \mathrm{U}$ between different groups. A Spearman ranks correlation test was applied to estimate the association between baseline VWF levels and BT200 concentrations needed to reduce VWF activity to $<20 \%$ of normal.

\section{Results}

In total, 33 LAA patients were included in the study whose baseline demographic data and clinical characteristics are given in Table 1 . Seventy-nine percent of patients suffered from stroke and $21 \%$ from transient ischemic attack and they were included on average 6 days after the event.

The median VWF activity level was 178\% (IQR: 103-253\%) and half of the patients had elevated levels (>180\%). Average concentrations of $1.3 \mu \mathrm{g} / \mathrm{ml} \mathrm{BT200}$ were needed to suppress VWF activity to $<20 \%$ of normal in this population (Fig. 1). The median VWF:RCo was 171\% (IQR: 57-285\%) and an average concentration of $2.5 \mu \mathrm{g} / \mathrm{ml}$ BT200 was needed to suppress VWF:RCo to $<20 \%$ of normal (Fig. 1). The baseline value of CADP-CT measured with PFA was $93 \mathrm{~s}$ (IQR: 50-136 s) and $1 \mu \mathrm{g} / \mathrm{ml}$ BT200 was needed to maximally prolong the CADP$\mathrm{CT}$ to $>300 \mathrm{~s}$ (Fig. 1). The median value of ristocetin induced aggregation was $40 \mathrm{U}$ (IQR: $10-79 \mathrm{U}$ ) and $1 \mu \mathrm{g} / \mathrm{ml}$ BT200 was needed to inhibit aggregation to $<20 \mathrm{U}$ (Fig. 1).

In a sensitivity analysis, we stratified patients into two groups: with normal ${ }^{29}$ (median: $150 \%$, range $67-178 \%$; $\mathrm{n}=17$ ) or elevated VWF activity (median: $221 \%$, range $184-423 \% ; n=16$ ). Significantly higher concentrations were needed to lower VWF activity to less than $20 \%$ of normal in patients with higher VWF activity levels (Suppl. fig 1), measured with REAADS ELISA, VWF:RiCo (Fig. 2; $\mathrm{p}<0.001$ ), or to inhibit VWF dependent platelet function. In an additional sensitivity analysis, we examined the difference between patients who received clopidogrel and those who did not receive it, and no significant difference was noticed in any of the four assays (Fig. 3; p >0.05).

BT200 did not alter clotting time as measured by thromboelastometry (not shown), indicating no unspecific effect on in vitro coagulation. 


\begin{tabular}{|c|c|}
\hline & Mean \pm SD, $[$ Median $\pm I Q R]$ or $n(\%)$ \\
\hline & $\mathrm{N}=33$ \\
\hline Age (years) & $72 \pm 10[73 \pm 16]$ \\
\hline Weight (kg) & $75 \pm 13[77.5 \pm 17]$ \\
\hline Gender (male) & $23(70 \%)$ \\
\hline \multicolumn{2}{|l|}{ Risk factors/past medical history n (\%) } \\
\hline Diabetes & $13(39)$ \\
\hline Hypertension & $23(70)$ \\
\hline Previous stroke & $13(39)$ \\
\hline Smoking & $18(54.5)$ \\
\hline Hypercholesterolemia & $19(58)$ \\
\hline Stenosis location left & $9(27)$ \\
\hline Stenosis location right & $15(45)$ \\
\hline Stenosis location both sides & $9(27)$ \\
\hline Stroke location left & $11(33)$ \\
\hline Stroke location right & $15(45)$ \\
\hline Transient ischemic attack & $7(21)$ \\
\hline Stenosis and stroke not on the same side & $1(33)$ \\
\hline \multicolumn{2}{|l|}{ Medication n (\%) } \\
\hline Aspirin & $23(70)$ \\
\hline Clopidogrel & $18(55)$ \\
\hline Statins & $18(55)$ \\
\hline Proton pump inhibitors & $9(27)$ \\
\hline Antidiabetics & $11(33)$ \\
\hline \multicolumn{2}{|l|}{ Laboratory data $($ mean \pm SD) $[$ median $\pm \mathrm{IQR}]$} \\
\hline Hemoglobin (g/dl) & $\begin{array}{l}13.1 \pm 2.21 \\
{[12.95 \pm 3.9]}\end{array}$ \\
\hline Platelets $(109 / \mathrm{L})$ & $\begin{array}{l}271 \pm 114 \\
{[250 \pm 122]}\end{array}$ \\
\hline Mean platelet volume (fL) & $\begin{array}{l}10.4 \pm 1.8 \\
{[10.2 \pm 1.5]}\end{array}$ \\
\hline Fibrinogen $(\mathrm{mg} / \mathrm{dl})$ & $\begin{array}{l}429 \pm 118 \\
{[404 \pm 166]}\end{array}$ \\
\hline Creatinine (mg/dl) & $\begin{array}{l}0.88 \pm 0.31 \\
{[0.79 \pm 0.38]}\end{array}$ \\
\hline High-sensitivity C-reactive protein $(\mathrm{mg} / \mathrm{L})$ & $\begin{array}{l}2.0 \pm 4.8 \\
{[0.39 \pm 1.55]}\end{array}$ \\
\hline \multicolumn{2}{|l|}{ VWF dependent assays $($ mean \pm SD) $[$ median $\pm \mathrm{IQR}]$} \\
\hline VWF activity REAADS ELISA (\%) & $\begin{array}{l}187 \pm 75 \\
{[178 \pm 75]}\end{array}$ \\
\hline Ristocetin cofactor activity (\%) & $\begin{array}{l}190 \pm 75 \\
{[171 \pm 114]}\end{array}$ \\
\hline Ristocetin induced aggregation (U) & $\begin{array}{l}50 \pm 36 \\
{[40 \pm 30]} \\
\end{array}$ \\
\hline Collagen adenosine diphosphate closure time (s) & $\begin{array}{l}116 \pm 74 \\
{[93 \pm 43]}\end{array}$ \\
\hline
\end{tabular}

Table 1. Demographic characteristics and baseline laboratory parameters. Data are reported as mean standard deviation (SD), median interquartile range (IQR) or $\mathrm{n}$ (number of patients) with percentages. VWF Von Willebrand Factor.

\section{Discussion}

Prevention of secondary strokes following an initial stroke is an area that has recently received attention since it is one of the few remaining frontiers in cardiovascular medicine where the existing standard of care is suboptimal $^{30}$. The complex nature of secondary stroke prevention requires consideration of the type and timing of the stroke. First, stroke prevention must be distinguished from the treatment of acute stroke. Second, the prevention of initial strokes must be distinguished from the prevention of subsequent secondary strokes. Apart from aggressive risk factor management (e.g., treatment of hypertension, hyperlipidemia, and/or diabetes), for the prevention of secondary strokes, the sub-type of a stroke suffered in the initial stroke must be considered in the selection of pharmacotherapy.

Prevention of secondary strokes after initial strokes due to Large Artery Atherosclerosis (LAA) requires antiplatelet therapy. The problem with the available therapeutic options is that the risk/benefit profile of the potent 


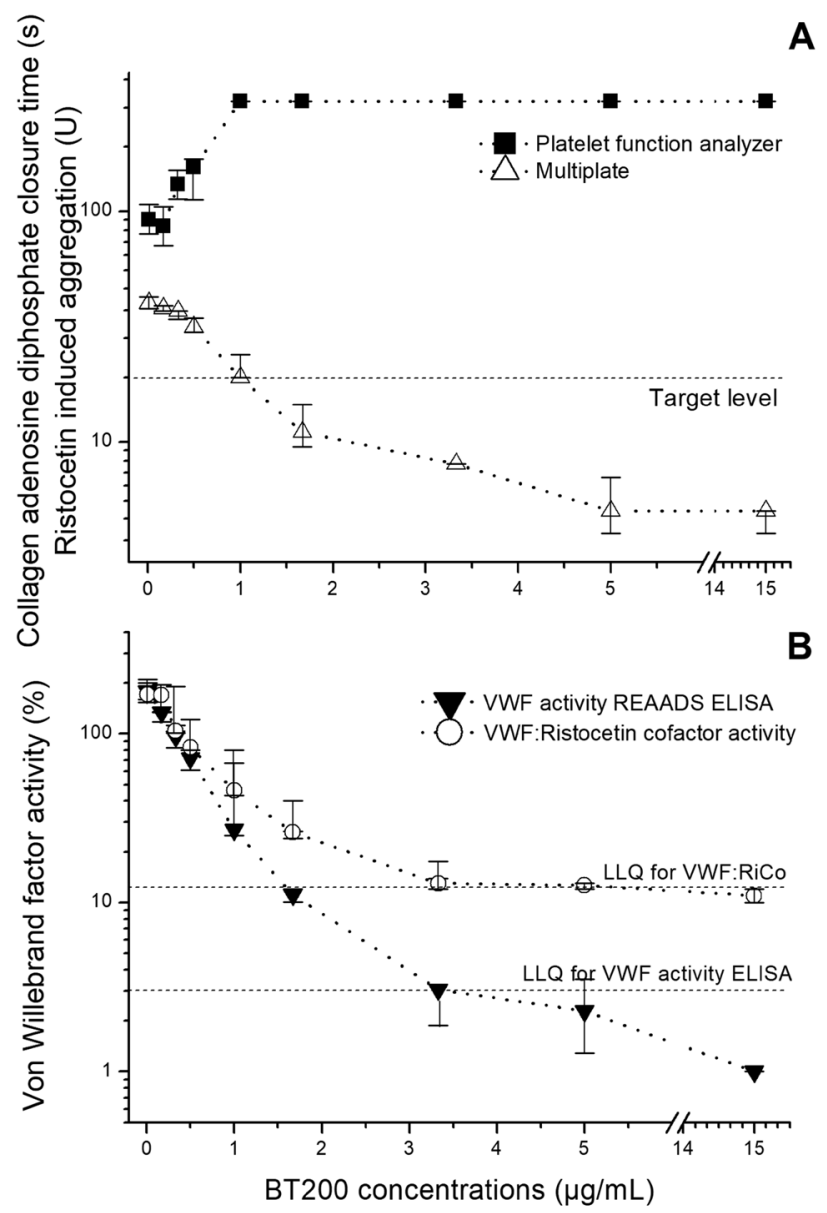

Figure 1. Concentration effect curves of the von Willebrand factor (VWF) inhibiting aptamer BT200 on platelet plug formation under high shear stress, ristocetin-induced whole blood aggregation (A), VWF activity and ristocetin cofactor activity $(\mathrm{RCo} ; \mathbf{B})$ in patients with large artery atherosclerosis stroke or transient ischemic attacks. BT200 was spiked ex vivo into plasma or whole blood. Collagen adenosine diphosphate closure time (CADP-CT) and aggregation were measured by the platelet function analyzer PFA-100 and Multiplate. Data are presented as median values and the interquartile range.

platelet inhibitors, so well- established in the domain of coronary disease, does not translate well into the domain of cerebrovascular disease. Aspirin in combination with clopidogrel reduces the number of recurrent strokes ${ }^{30}$ but does not prevent $75 \%$ of recurrent events. Ticagrelor, non-significantly reduced the primary endpoint by only $11 \%$ in the SOCRATES trial, a secondary stroke prevention study with $>13,000$ stroke/TIA patients ${ }^{31}$, and in the THALES trial the combination of ticagrelor with aspirin decreased the risk of composite of stroke or death within 30 days, but the incidence of disability did not differ ${ }^{32}$. Other potent platelet inhibitors indicated for use in patients with acute coronary syndromes such as prasugrel or vorapaxar carry a black box warning and are contra-indicated in stroke patients because of bleeding risk. Improved secondary stroke prevention requires new approaches to platelet function inhibition other than the $\mathrm{n}^{\text {th }}$ generation ADP/P2Y12 inhibitors. Thus, there is an unmet medical need for novel anti-thrombotic pharmacotherapy with an improved risk/benefit profile for use in secondary stroke prevention.

The stroke sub-type that we are selectively targeting is LAA. Published medical literature strongly implicates VWF to the pathophysiologic mechanism of LAA stroke, most likely because platelet thrombus formation in the setting of atherosclerotic plaque has an important shear-dependent component that is mediated by VWF. No difference in the inhibitory BT200 concentrations was seen in VWF dependent platelet function tests between the patients with or without clopidogrel. This indicates that clopidogrel is suboptimally effective when the pathogenesis of thrombus formation is driven by increased VWF activity. Additionally, it was shown that VWF inhibition by another anti-VWF aptamer called ARC1779 significantly reduces thromboembolism (a strong predictor of stroke) in patients after carotid endarterectomy ${ }^{33}$ while other antiplatelet drugs including clopidogrel failed to show that effect ${ }^{34}$.

Numerous epidemiologic studies have established that an excess of VWF indicates an increased risk of stroke $^{35}$, and stroke mortality ${ }^{36}$, but even patients with normal VWF levels can have first or recurrent LAA strokes; conversely, a relative deficiency of VWF may be protective ${ }^{29,37}$. Patients with average VWF plasma levels 

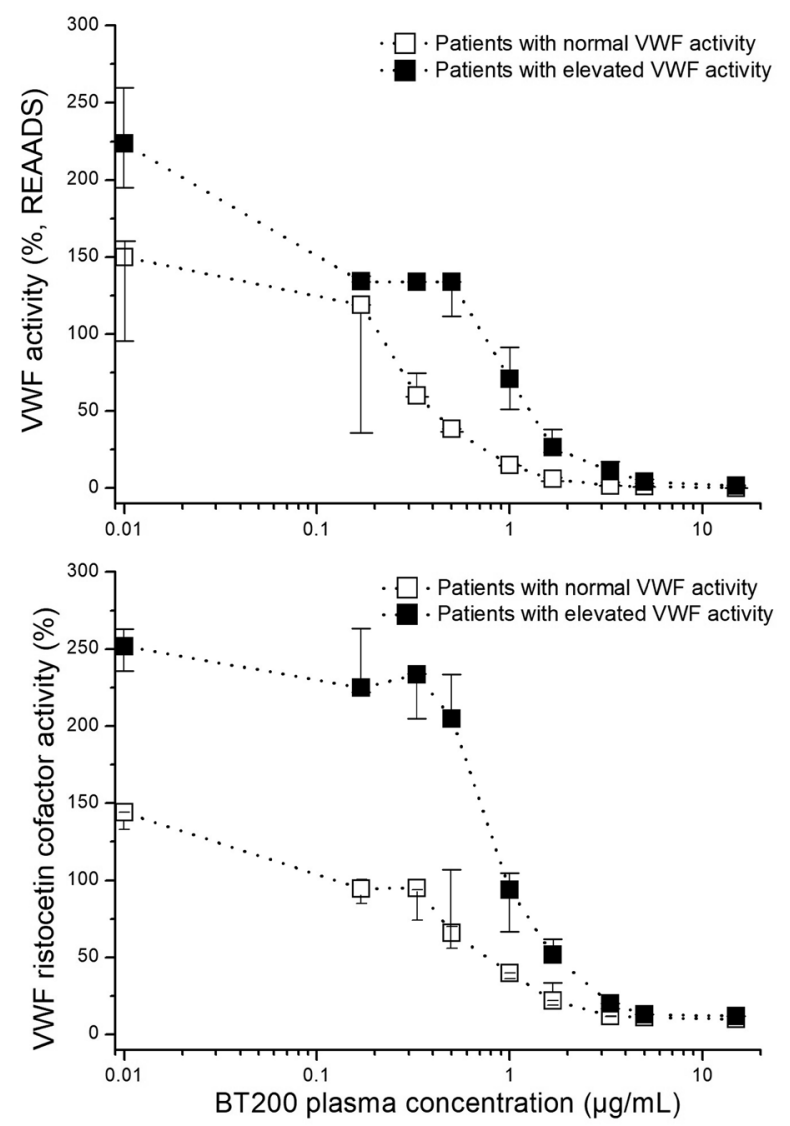

Figure 2. Target concentration dependent effects of BT200 on von Willebrand factor (VWF) activity (A) and VWF ristocetin cofactor activity $(\mathbf{B})$ in patients with large artery atherosclerosis stroke or transient ischemic attacks when stratified into groups with normal $(<180 \%)$ or elevated $(>180 \%)$ VWF activity. Data are presented as median values and interquartile ranges and baseline is presented as 0.01 for better visualization on the log scale.

of $24 \%$ had a $35-67 \%$ reduced risk for ischemic stroke as compared to controls, suggesting that partial inhibition of VWF could be protective in the stroke-prone population ${ }^{29}$.

Findings from this study are essential for planning of trials with VWF inhibitors such as BT200 because our previous experiments did not provide insight into the effects of BT200 on platelet function in any patients with atherosclerotic disease. For the first time, we were able to examine BT200 effects in patients with chronically elevated VWF, which may not be the same as newly released VWF such as seen after desmopressin or endotoxin administration ${ }^{38}$. Freshly released VWF is ultra-large and hyperactive but under normal conditions, it is rapidly degraded by ADAMTS13 which prevents micro thrombosis ${ }^{39}$. There is a marked difference in the levels of VWF and VWF-propeptide in healthy individuals after desmopressin/endotoxin when compared to patients with diabetes, thrombotic thrombocytopenic purpura (TTP), or sepsis ${ }^{40}$. This results from differences in altered secretion but also de novo synthesis of VWF due to enhanced transcriptional activity ${ }^{40}$.

For this reason, it was of interest to investigate the effects of BT200 on platelet function not only in healthy individuals after stimulated VWF release, which we did in a previous study ${ }^{38}$ but also in a patient group with chronically activated endothelium. The patient population in our study had a mean of age of 72 years and different comorbidities, which are all known to enhance VWF levels ${ }^{41}$. In such a population the endothelium is chronically activated and chronic inflammation is present, which is influencing platelet function ${ }^{42-44}$. Importantly, however, there are also changes in platelet function associated with aging ${ }^{45,46}$, but very limited information is available in an elderly population ${ }^{47}$. This was a relevant shortcoming of our previous study ${ }^{38}$ testing platelet function in healthy volunteers. The reason why we chose this specific subset of stroke patients, is because LAA stroke patients represent the lead target indication for BT200, for obvious reasons which were recently reviewed ${ }^{35}$.

As mentioned, in secondary stroke prevention of LAA stroke, patients are treated either with aspirin, aspirin plus dypiridamole, or clopidogrel ${ }^{48}$ or a combination thereof, while ticagrelor use will likely become more common ${ }^{32}$. Patients in our study were treated with aspirin or clopidogrel, which allowed us to investigate possible interactions of BT200 with those antiplatelet drugs. Clopidogrel and other potent P2Y12 inhibitors such as prasugrel have been proven to inhibit not only platelet activation and aggregation but prasugrel also reduced levels of thromboxane $\mathrm{A} 2^{49}$ and both prasugrel and clopidogrel reduced platelet interaction with monocytes and leukocytes in mice ${ }^{49,50}$. This is important because platelet-leukocyte aggregates play a major role and modulate 

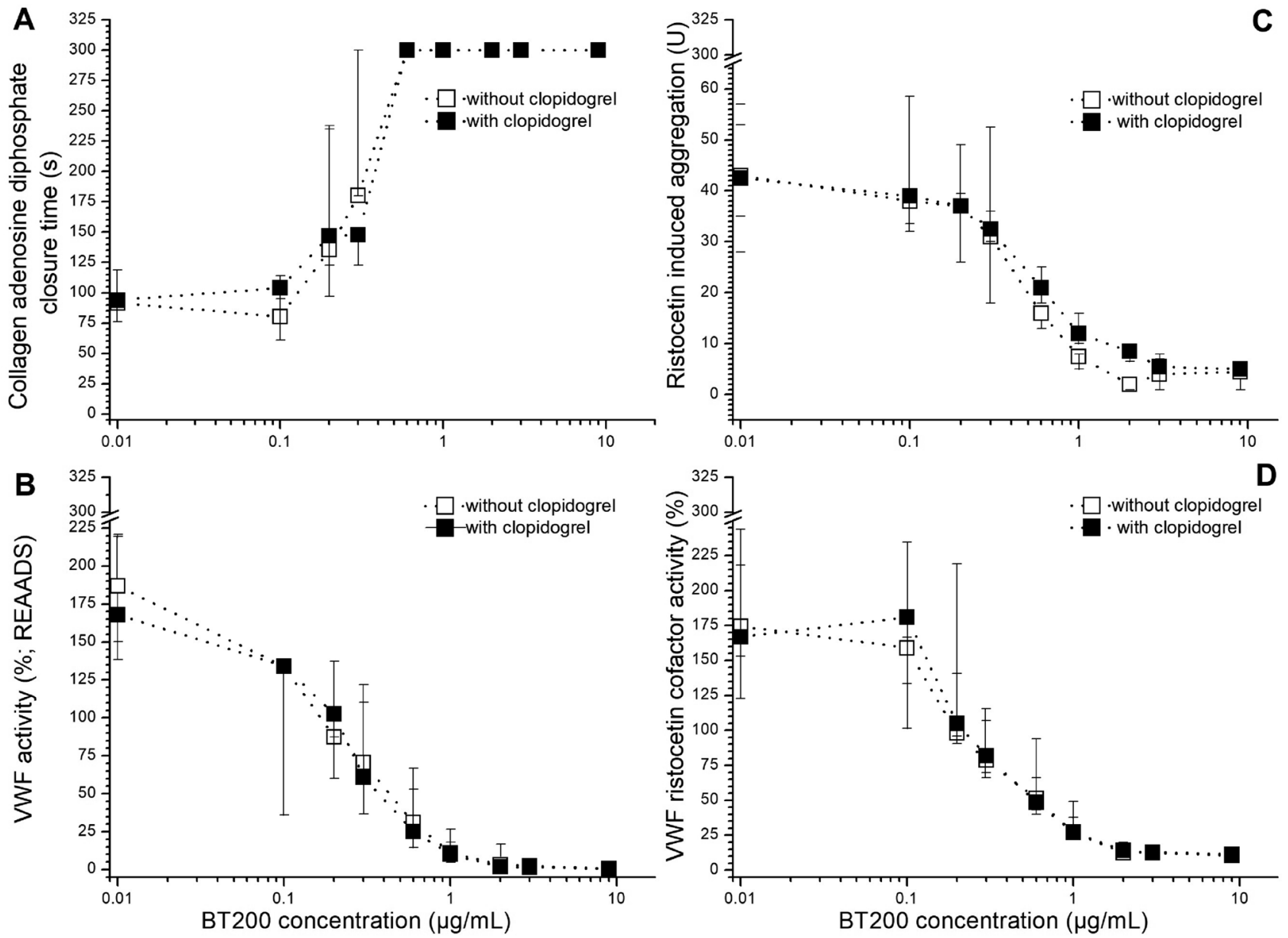

Figure 3. Concentration effect curves of BT200 on platelet plug formation under high shear stress (A), ristocetin-induced platelet aggregation (B), von Willebrand Factor (VWF) activity $(\mathbf{C})$ and ristocetin cofactor activity (VWF:RCo) (D) in patients with large artery atherosclerosis stroke or transient ischemic attacks with $(n=18)$ or without clopidogrel therapy $(n=15)$. Data are presented as median values and the interquartile range, and baseline is presented as 0.01 for better visualization on the log scale.

the development and progression of vascular diseases ${ }^{51}$. In a study with another anti-VWF aptamer ARC15105, this aptamer reduced the aggregation of platelets induced by a number of different agonists including ristocetin, collagen, adenosine diphosphate, and thrombin receptor activating peptide. This possibly reflects outside/ in signalling and this inhibitory effect of ARC15105 was significant both in healthy individuals but also in myocardial infarction patients who were all pretreated with aspirin, clopidogrel, and heparin ${ }^{52}$. Such a possible synergist effect can only be seen in platelet function tests applied to blood of the target population. Therefore, it was of interest to characterize whether the effect of BT200 would be synergistic and/or specific when used in combination with aspirin or clopidogrel. In our study, the intake of other antiplatelet drugs did not influence in vitro effects of BT200.

It is important to mention that some assays such as the VWF ELISA and RiCo tests, which are used to measure VWF activity, usually require high dilution (up to 20-fold) of the samples. High dilution of samples could influence the binding of the aptamer to VWF and can wash the aptamer of its target. This could lead to an overestimation of the doses of BT200 needed for the appropriate VWF inhibition, which may lead to undue interference with the safety of the drug. In contrast platelet function tests performed in fresh blood usually do not require dilution or only minimal dilution, which provides more accurate estimates for concentration-effect curves, and therefore subsequent dosing. In addition, the PFA assay allows measuring VWF function under high shear rates, which is the physiological activator inducing conformational change and therefore activation of VWF. We have noticed that slightly higher concentrations of BT200 are needed for the expected effect when using tests with a high dilution of the samples when compared to the whole blood tests without or with a low dilution of the sample. This is important for estimating the effective dose of BT200 that will be used in clinical trials and it is essential that these estimations are correct. For all of the mentioned reasons, this study delivered important insights for planning future clinical trials with VWF inhibitors.

In this study, we have stratified our population into two groups, patients with VWF activity below or above $180 \%$. Similar to previous studies where we investigated ex vivo effects of BT200 in healthy volunteers after stimulated VWF release with endotoxin or desmopressin ${ }^{38}$ and in acute coronary syndrome (ACS) patients $\mathrm{s}^{53}$, 
we have again shown that higher concentrations of BT200 were needed for the same effect in cases of increased VWF when compared to normal VWF levels.

In all three studies of our group, BT200 inhibited VWF in a target-concentration dependent manner. Similarly, another VWF inhibitor called caplacizumab (anti-VWF antibody) was characterized by a target-mediated disposition and the exposure was dependent upon drug and target concentration over time ${ }^{54}$. This suggests that this effect could be a class effect of anti-VWF agents, this finding is important for further insights in pharmacokinetics and effects of BT200.

Finally, it is important to mention that a specific antidote for BT200 has been synthesized and evaluated ${ }^{55}$. Because BT200 is an aptamer, synthesis of an antidote is a rather simple procedure. Inhibition of BT200 in a monkey model has been effective and safe and it is yet to be evaluated in humans. Having a highly specific, effective, and nontoxic antidote is beneficial and it is an advantage especially in cases of any adverse events, such as bleeding or in cases of emergency procedures.

\section{Conclusion}

The anti-VWF aptamer BT200 effectively inhibited VWF activity even in the presence of high VWF levels found in LAA stroke patients, in a target concentration-dependent manner. Together with a long half-life supporting once-weekly administration, and the existence of a reversal agent, BT200 is an interesting drug candidate for secondary prevention of LAA stroke as well as rarer stroke entities with an unmet medical need.

\section{Limitations}

The number of patients included in the study was limited but provides good precision to estimate the variability of effective levels of the VWF inhibitor. Additionally, concentration-effect curves were established ex vivo. Nonetheless, effective concentrations may be directly extrapolated to in vivo application, because BT200 inhibits a plasma protein ${ }^{10}$. So far it has not been determined which are exact levels of VWF inhibition that could prevent arterial thrombosis. In the epidemiologic data, it is shown that patients who suffered from von Willebrand disease with VWF activity of about $25 \%{ }^{29}$, had approximately $40-60 \%$ fewer arterial thrombotic events, which is why we assumed that decreasing VWF activity to $20 \%$ or less is of therapeutic interest.

This study cannot provide estimates about bleeding risks associated with BT200, but von Willebrand disease is characterized by sporadic mucocutaenous bleedings which are not spontaneous except for exaggerated menstrual bleedings, a condition rarely prevalent in LAA stroke patients. Nevertheless, considering the potential for bleeding caused by trauma or surgery, a specific reversal agent for BT200 has been created and successfully tested in nonhuman primates ${ }^{55}$.

Received: 13 July 2020; Accepted: 25 January 2021

Published online: 04 February 2021

\section{References}

1. Benjamin, E. J. et al. Heart disease and stroke statistics-2019 update: A report from the American Heart Association. Circulation 139, e56-e528 (2019).

2. Feigin, V. L., Norrving, B. \& Mensah, G. A. Global burden of stroke. Circ. Res. 120, 439-448 (2017).

3. Duke, R. J., Bloch, R. F., Turpie, A. G., Trebilcock, R. \& Bayer, N. Intravenous heparin for the prevention of stroke progression in acute partial stable stroke. Ann. Intern. Med. 105, 825-828 (1986).

4. The International Stroke Trial (IST): A randomised trial of aspirin, subcutaneous heparin, both, or neither among 19435 patients with acute ischaemic stroke. International Stroke Trial Collaborative Group. Lancet 349, 1569-1581 (1997).

5. Adams, H. P. Jr. et al. Emergency administration of abciximab for treatment of patients with acute ischemic stroke: Results of an international phase III trial: Abciximab in emergency treatment of stroke trial (AbESTT-II). Stroke 39, 87-99 (2008).

6. Kellert, L. et al. Endovascular stroke therapy: Tirofiban is associated with risk of fatal intracerebral hemorrhage and poor outcome. Stroke 44, 1453-1455 (2013).

7. Siebler, M. et al. Safety of tirofiban in acute Ischemic Stroke: The SaTIS trial. Stroke 42, 2388-2392 (2011).

8. Serebruany, V. L., Alberts, M. J. \& Hanley, D. F. Prasugrel in the poststroke cohort of the TRITON Trial: The clear and present danger. Cerebrovasc. Dis. 26, 93-94 (2008).

9. Morrow, D. A. et al. Efficacy and safety of vorapaxar in patients with prior ischemic stroke. Stroke 44, 691-698 (2013).

10. Zhu, S. et al. The development and characterization of a long acting anti-thrombotic von Willebrand factor (VWF) aptamer. J. Thromb. Haemostas. JTH 18, 1113-1123 (2020).

11. Reininger, A. J. Function of von Willebrand factor in haemostasis and thrombosis. Haemophilia 14(Suppl 5), 11-26 (2008).

12. Li, M. et al. Enhanced shear-induced von Willebrand factor binding to platelets in acute myocardial infarction. Thromb. Res. 100, 251-261 (2000).

13. Ruggeri, Z. M. \& von Ware, J. Willebrand factor. FASEB J. 7, 308-316 (1993).

14. Reininger, A. J. et al. Mechanism of platelet adhesion to von Willebrand factor and microparticle formation under high shear stress. Blood 107, 3537-3545 (2006).

15. Fasipe, T. A. et al. Extracellular Vimentin/VWF (von Willebrand factor) interaction contributes to VWF string formation and stroke pathology. Stroke 49, 2536-2540 (2018).

16. Derhaschnig, U., Pachinger, C. \& Jilma, B. Variable inhibition of high-shear-induced platelet plug formation by eptifibatide and tirofiban under conditions of platelet activation and high von Willebrand release: A randomized, placebo-controlled, clinical trial. Am. Heart J. 147, E17 (2004).

17. Reiter, R. A. et al. Desmopressin antagonizes the in vitro platelet dysfunction induced by GPIIb/IIIa inhibitors and aspirin. Blood 102, 4594-4599 (2003).

18. Spiel, A. O. et al. Effects of prasugrel on platelet inhibition during systemic endotoxaemia: A randomized controlled trial. Clin. Sci. 123, 591-600 (2012).

19. De Meyer, S. F., Stoll, G., Wagner, D. D. \& von Kleinschnitz, C. Willebrand factor: An emerging target in stroke therapy. Stroke 43, 599-606 (2012). 
20. Williams, S. R. et al. Genetic drivers of von Willebrand factor levels in an ischemic stroke population and association with risk for recurrent stroke. Stroke 48, 1444-1450 (2017).

21. Carter, A. M., Catto, A. J., Mansfield, M. W., Bamford, J. M. \& Grant, P. J. Predictive variables for mortality after acute ischemic stroke. Stroke 38, 1873-1880 (2007).

22. Greisenegger, S. et al. Biomarkers and mortality after transient ischemic attack and minor ischemic stroke. Stroke 46, 659-666 (2015).

23. Jilma, B. Platelet function analyzer (PFA-100): A tool to quantify congenital or acquired platelet dysfunction. J. Lab. Clin. Med. 138, 152-163 (2001)

24. Toth, O., Calatzis, A., Penz, S., Losonczy, H. \& Siess, W. Multiple electrode aggregometry: A new device to measure platelet aggregation in whole blood. Thromb. Haemost. 96, 781-788 (2006).

25. Schmidt, D. E. et al. Whole blood ristocetin-activated platelet impedance aggregometry (Multiplate) for the rapid detection of Von Willebrand disease. Thromb. Haemost. 117, 1528-1533 (2017).

26. Ewenstein, B. M. Use of ristocetin cofactor activity in the management of von Willebrand disease. Haemophilia 7, 10-15 (2001).

27. Murdock, P. J., Woodhams, B. J., Matthews, K. B., Pasi, K. J. \& von Goodall, A. H. Willebrand factor activity detected in a monoclonal antibody-based ELISA: An alternative to the ristocetin cofactor platelet agglutination assay for diagnostic use. Thromb. Haemost. 78, 1272-1277 (1997).

28. Spiel, A. O., Mayr, F. B., Firbas, C., Quehenberger, P. \& Jilma, B. Validation of rotation thrombelastography in a model of systemic activation of fibrinolysis and coagulation in humans. J. Thromb. Haemostas. JTH 4, 411-416 (2006).

29. Sanders, Y. V. et al. Reduced prevalence of arterial thrombosis in von Willebrand disease. J. Thromb. Haemostas. JTH 11, 845-854 (2013).

30. Johnston, S. C. et al. Clopidogrel and aspirin in acute ischemic stroke and high-risk TIA. N. Engl. J. Med. 379, 215-225 (2018).

31. Johnston, S. C. \& Amarenco, P. Ticagrelor versus aspirin in acute stroke or transient ischemic attack. N. Engl. J. Med. 375, 1395 (2016).

32. Johnston, S. C. et al. Ticagrelor and aspirin or aspirin alone in acute ischemic stroke or TIA. N. Engl. J. Med. 383, 207-217 (2020).

33. Markus, H. S. et al. The von Willebrand inhibitor ARC1779 reduces cerebral embolization after carotid endarterectomy: A randomized trial. Stroke 42, 2149-2153 (2011).

34. de Borst, G. J. et al. Influence of antiplatelet therapy on cerebral micro-emboli after carotid endarterectomy using postoperative transcranial Doppler monitoring. Eur. J. Vasc. Endovasc. Surg. 34, 135-142 (2007).

35. Buchtele, N., Schwameis, M., Gilbert, J. C., Schorgenhofer, C. \& Jilma, B. Targeting von Willebrand factor in ischaemic stroke: focus on clinical evidence. Thromb. Haemost. 118, 959-978 (2018).

36. Catto, A. J. et al. von Willebrand factor and factor VIII: C in acute cerebrovascular disease. Relationship to stroke subtype and mortality. Thromb. Haemostas. 77, 1104-1108 (1997).

37. Seaman, C. D., Yabes, J., Comer, D. M. \& Ragni, M. V. Does deficiency of von Willebrand factor protect against cardiovascular disease? Analysis of a national discharge register. J. Thromb. Haemost. JTH 13, 1999-2003 (2015).

38. Kovacevic, K. D. et al. The aptamer BT200 effectively inhibits von Willebrand factor (VWF) dependent platelet function after stimulated VWF release by desmopressin or endotoxin. Sci. Rep. 10, 11180-11180 (2020).

39. Kawecki, C., Lenting, P. J. \& von Denis, C. V. Willebrand factor and inflammation. J. Thromb. Haemost. JTH 15, 1285-1294 (2017).

40. Van Mourik, J. \& Romani, T. Von Willebrand Factor Propeptide in Vascular Disorders, (2001).

41. Blann, A. D. Plasma von Willebrand factor, thrombosis, and the endothelium: the first 30 years. Thromb. Haemost. 95, 49-55 (2006).

42. Stokes, K. Y. \& Granger, D. N. Platelets: A critical link between inflammation and microvascular dysfunction. J. Physiol. 590, 1023-1034 (2012).

43. Bakogiannis, C., Sachse, M., Stamatelopoulos, K. \& Stellos, K. Platelet-derived chemokines in inflammation and atherosclerosis. Cytokine 122, 154157 (2019).

44. Nurden, A. T. Platelets, inflammation and tissue regeneration. Thromb. Haemost. 105(Suppl 1), S13-33 (2011).

45. Gleerup, G. \& Winther, K. The effect of ageing on platelet function and fibrinolytic activity. Angiology 46, 715-718 (1995).

46. Davis, P. B. \& Silski, C. Ageing and the alpha 2-adrenergic system of the platelet. Clin. Sci. 73, 507-513 (1987).

47. Jones, C. I. Platelet function and ageing. Mammal. Genome 27, 358-366 (2016).

48. Hackam, D. G. \& Spence, J. D. Antiplatelet therapy in ischemic stroke and transient ischemic attack. Stroke 50, 773-778 (2019).

49. Totani, L. et al. Prasugrel inhibits platelet-leukocyte interaction and reduces inflammatory markers in a model of endotoxic shock in the mouse. Thromb. Haemost. 107, 1130-1140 (2012).

50. Evangelista, V. et al. Clopidogrel inhibits platelet-leukocyte adhesion and platelet-dependent leukocyte activation. Thromb. Haemost. 94, 568-577 (2005).

51. Schrottmaier, W. C., Mussbacher, M., Salzmann, M. \& Assinger, A. Platelet-leukocyte interplay during vascular disease. Atherosclerosis 307, 109-120 (2020).

52. Siller-Matula, J. M. et al. ARC15105 is a potent antagonist of von Willebrand factor mediated platelet activation and adhesion. Arterioscler. Thromb. Vasc. Biol. 32, 902-909 (2012).

53. Kovacevic, K.D., et al. von Willebrand factor predicts mortality in ACS patients treated with potent P2Y12 antagonists and is inhibited by aptamer BT200 ex vivo. Thromb. Haemost. (2020).

54. Sargentini-Maier, M. L. et al. Clinical pharmacology of caplacizumab for the treatment of patients with acquired thrombotic thrombocytopenic purpura. Exp. Rev. Clin. Pharmacol. 12, 537-545 (2019).

55. Zhu, S., et al. Potent and rapid reversal of the von Willebrand Factor Inhibitor Aptamer BT200. J. Thromb. Haemost JTH (2020).

\section{Author contributions}

K.D.K. wrote a draft of the paper, performed analysis, and did a statistical evaluation of the results. S.G. from the department of Neurology provided patients for the study. A.L. helped in providing samples from the patients. G.G. and N.B. included patients in the study, took informed consent, and drew samples. I.P. contributed to the idea of the study. K.P. helped in the performance of the analysis. S.Z., J.C.G., and B.J. developed the idea of the study and conducted the study. B.J. wrote parts of the paper and revised it.

\section{Funding}

This trial was funded by the Austrian Science Funds, Grant\#: SFB54-P04.

\section{Competing interests}

Bernd Jilma, Shuhao Zhu and James C. Gilbert are cofounders of Band Therapeutics, SZ and JCG are employees of and BJ a consultant to Guardian Therapeutics. All other authors declare that they have no relevant conflict of interest. 


\section{Additional information}

Supplementary Information The online version contains supplementary material available at https://doi. org/10.1038/s41598-021-82747-7.

Correspondence and requests for materials should be addressed to K.D.K.

Reprints and permissions information is available at www.nature.com/reprints.

Publisher's note Springer Nature remains neutral with regard to jurisdictional claims in published maps and institutional affiliations.

(c) (1) Open Access This article is licensed under a Creative Commons Attribution 4.0 International cc) License, which permits use, sharing, adaptation, distribution and reproduction in any medium or format, as long as you give appropriate credit to the original author(s) and the source, provide a link to the Creative Commons licence, and indicate if changes were made. The images or other third party material in this article are included in the article's Creative Commons licence, unless indicated otherwise in a credit line to the material. If material is not included in the article's Creative Commons licence and your intended use is not permitted by statutory regulation or exceeds the permitted use, you will need to obtain permission directly from the copyright holder. To view a copy of this licence, visit http://creativecommons.org/licenses/by/4.0/.

(C) The Author(s) 2021 